\title{
Florecimientos algales nocivos en las costas de Campeche, Golfo de México
}

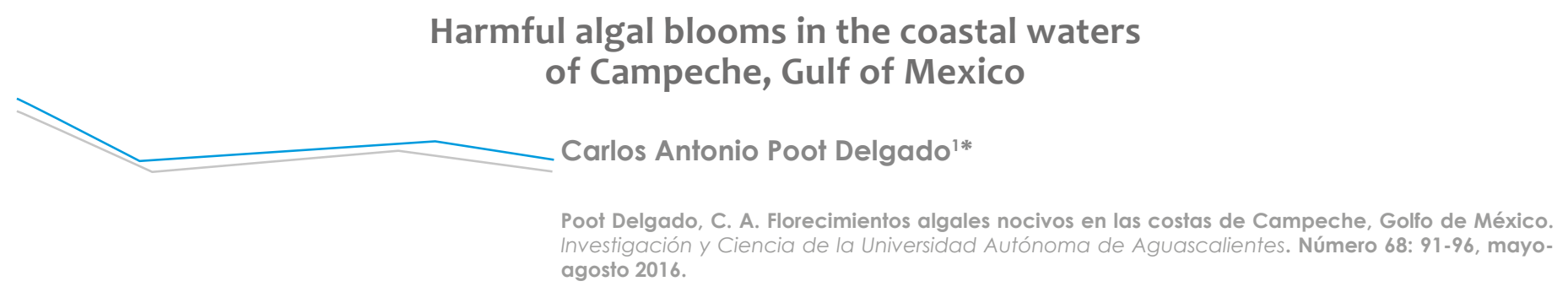

\section{RESUMEN}

El presente estudio describe el estado de conocimiento sobre florecimientos algales nocivos (FAN) en las aguas costeras del estado de Campeche, México. Se realizó una búsqueda bibliográfica de 1965 a 2014 consultando reportes oficiales, reuniones académicas y artículos especializados (aproximadamente 30). El análisis de la literatura mostró que ha habido un avance significativo en los reportes de FAN. La abundancia de las especies nocivas presentó valores altos $\left(10^{6}\right.$ células $\left.\right|^{-1}$ ) durante la temporada de lluvias (junioseptiembre). Se registró la presencia de especies potencialmente nocivas: los dinoflagelados Karenia brevis, Pyrodinium bahamense var. bahamense, Prorocentrum mexicanum, P. minimum y la cianobacteria Cylindrospermopsis cuspis.

\section{ABSTRACT}

The present study describes the state of knowledge of harmful algal blooms (HAB) in the coastal waters of the State of Campeche, Mexico. A bibliography on the subject was compiled during the period from 1965 to 2014 and considers official reports, scientific meetings and specialized articles (approximately 30). An analysis of the literature showed significant advances

Palabras clave: Campeche, dinoflagelados, florecimientos algales nocivos, Golfo de México, microalgas. Keywords: Campeche, dinoflagellates, Gulf of Mexico, harmful algal blooms, microalgae. in the reports of $\mathrm{HAB}$. The abundance of noxious species was the highest $\left(10^{6} \mathrm{Cells}^{-1}\right)$ in the rainy season (June-September). The following potentially harmful species were reported: the dinoflagellates Karenia brevis, Pyrodinium bahamense var. bahamense, Prorocentrum mexicanum and $P$. minimum, and the cyanobacterium Cylindrospermopsis cuspis.

\section{INTRODUCCIÓN}

Más de $70 \%$ de la población humana reside dentro de los primeros $100 \mathrm{~km}$ de la zona costera (Paerl et al., 2006), lo que da como resultado más evidente el enriquecimiento de nutrientes (nitrógeno, fósforo y silicato), y una variedad de micronutrientes (metales traza y vitaminas) diluidos en el medio costero (Anderson et al., 2012). Ello ocasiona cambios en la producción primaria, clorofila a y la abundancia celular (Bricker et al., 2003), evidenciado por el aumento de los florecimientos algales nocivos (FAN) (Glibert et al., 2005), que producen respuestas tales como hipoxia y anoxia en la columna de agua (Kaas et al., 2005).

Los FAN consisten en un crecimiento explosivo de organismos fotosintéticos, mixótrofos o heterótrofos susceptibles de afectar a otros organismos (incluyendo al hombre) que utilizan el ambiente acuático. Pueden causar intoxicaciones a través de la producción de sustancias químicas (toxinas), alterando la fisiología de los organismos afectados (Smayda, 1997).

Los síndromes tóxicos más conocidos causados por fitoplancton son: el PSP (intoxicación paralítica de los bivalvos), el DSP (intoxicación diarreica de los bivalvos), el ASP (intoxicación amnésica de los 
bivalvos) y el NSP (intoxicación neurotóxica de los bivalvos) (Sar et al., 2002).

En México, en una reciente revisión sobre los FAN (Band Schmidt et al., 2011) se observa un incremento en los reportes de especies nocivas; así como sus efectos en los cultivos de camarón (Alonso Rodríguez y Páez Osuna, 2003) y en organismos silvestres (Núñez Vázquez et al., 2011; Gárate Lizárraga y González Armas, 2014).

Dado que los FAN representan una seria amenaza se requiere describir la información básica sobre las especies causantes en diversas regiones, hábitats y diferentes épocas del año. Esta revisión da a conocer el estado de conocimiento de los estudios de FAN en el ámbito marino en las costas campechanas, con el objetivo de analizar los reportes cada vez más frecuentes sobre las especies causantes.

\section{Diatomeas y dinoflagelados nocivos}

En estudios realizados en el banco de Campeche, autores rusos reportaron FAN de dinoflagelados planctónicos desde julio de 1965, en donde observaron y registraron una coloración en el agua de mar producida por el dinoflagelado Scrippsiella trochoidea reportado como Gonyaulax minima y Karenia brevis reportado como Gymnodinium breve (Okolodkov, 2003).

Desde 2003 la Comisión para la Protección contra Riesgos Sanitarios del estado de Campeche (COPRISCAM) integró el Proyecto Nacional de Marea Roja bajo la coordinación de la Comisión Federal para la Protección contra Riesgos Sanitarios (COFEPRIS) realizando monitoreos a lo largo del litoral campechano (Figura 1).

La COPRISCAM registró florecimientos de Pyrodinium bahamense en septiembre de 2005, en la bahía de Campeche y de K. brevis en Frontera, Tabasco y Nuevo Campechito. Para 2006, Peridinium quinquecorne se reportó en la bahía de Campeche. En junio y septiembre de 2008 Rhizosolenia y Chaetoceros estuvieron presentes, al igual que Pseudonitzschia spp., en la región norte del litoral del estado de Campeche (Tabla 1).

Ese año en la zona de los Petenes y la bahía de Campeche los dinoflagelados nocivos: P. bahamense, Prorocentrum mexicanum, Prorocentrum minimum, Prorocentrum hoffmanianum, Gymnodinium

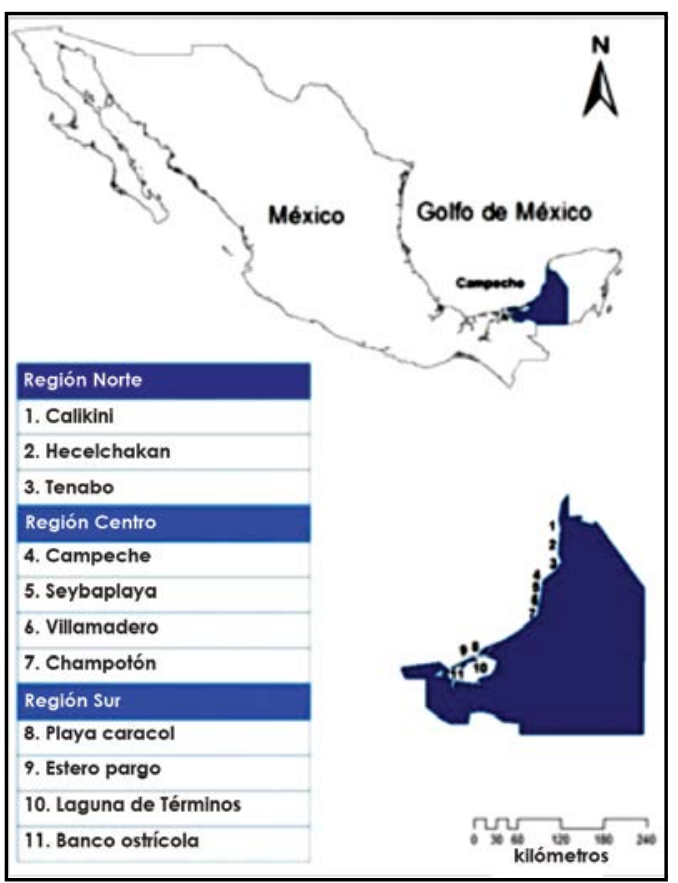

Figura 1. Litoral del estado de Campeche con reportes de FAN. Los números indican sitios con reportes de FAN de 2003-2014. Mapa elaborado por Jorge A. Ortiz Lozano.

catenatum y Gambierdiscus estuvieron presentes en la época de lluvias (junio-octubre), con rangos de abundancias de 100 a $26 \times 10^{4}$ células ${ }^{-1}$ (Poot Delgado y Guzmán Noz, 2010).

El dinoflagelado nocivo Prorocentrum micans fue observado en el área de extracción de ostión en la Laguna de Términos en mayo de 2010; en el 2011 se observó a K. brevis en la costa de Calkini, Hecelchakan y Campeche (Soto Ramos et al., 2012; Del Ángel Tafoya, 2013).

En las aguas costeras de Champotón en el centro del estado de Campeche, de mayo de 2010 a noviembre de 2011 Poot Delgado y Rosado García (2013) reportan la presencia de Gambierdiscus, G. catenatum, K. brevis, P. bahamense, P. mexicanum, $P$. minimum y $P$. hoffmannianum, con abundancia

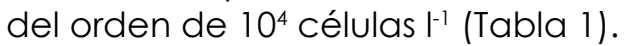

En noviembre de 2013 se registró a la diatomea Rhizosolenia con una abundancia máxima de $1.8 \mathrm{x}$ $10^{5}$ células $^{1-1}$, que puede estar asociada a la mortandad de peces y mantarrayas, probablemente por la disminución de las concentraciones normales de oxígeno disuelto (Rendón von Osten et al., 2013). 
Tabla 1. Especies causantes de florecimientos algales nocivos en las costas de Campeche

\begin{tabular}{|c|c|c|c|c|}
\hline Especie & Localidad & Fecha & $\begin{array}{l}\text { Abundancia } \\
\text { máxima } \\
\left(\text { células } I^{-1}\right)\end{array}$ & Referencias \\
\hline \multirow{3}{*}{ Pyrodinium bahamense var. bahamense' } & $\begin{array}{l}\text { Costa Villamar, } \\
\text { Champotón }\end{array}$ & 20 de marzo de 2005 & 30,000 & \multirow{3}{*}{ COPRISCAM y LESP* } \\
\hline & \multirow{2}{*}{ Malecón de Campeche } & \multirow{2}{*}{9 de agosto de 2005} & 330,000 & \\
\hline & & & 315,000 & \\
\hline Scrippsiella spp. ${ }^{5}$ & Banco de Campeche & 24 de agosto de 2005 & 300,000 & Soto Ramos et al. (2012) \\
\hline Pseudo-nitzschia sp.' & Isla Arena, Calkini & $\begin{array}{c}8 \text { de diciembre del } \\
2005\end{array}$ & 30,000 & \multirow{2}{*}{ COPRISCAM Y LESP* } \\
\hline Prorocentrum compressum?! & $\begin{array}{l}\text { Nvo. Campechito, Cd. } \\
\text { Del Carmen }\end{array}$ & 11 al 17 de abril 2007 & 185,000 & \\
\hline Anabaena sp. ${ }^{3}$ & \multirow{2}{*}{ Bahía de Campeche } & Diciembre de 2007 & 323,000 & $\begin{array}{c}\text { Poot Delgado y Guzmán } \\
\text { Noz (2010) }\end{array}$ \\
\hline Pyrodinium bahamense var. bahamense & & Abril de 2008 & 80,000 & Poot Delgado et al. (2009) \\
\hline Pseudo-nitzschia sp.' & \multirow{3}{*}{$\begin{array}{l}\text { Región norte litoral (costa } \\
\text { de Calkini-Hecelchakan) }\end{array}$} & \multirow{3}{*}{$\begin{array}{c}26 \text { junio al } 05 \\
\text { septiembre de } 2008\end{array}$} & 450,000 & \multirow{3}{*}{ COPRISCAM Y LESP* } \\
\hline Rhizosolenia sp. $^{5}$ & & & 360,000 & \\
\hline Chaetoceros sp. ${ }^{4}$ & & & 260,000 & \\
\hline Pseudo-nitzschia spp.' & Bahía de Campeche & Diciembre de 2008 & 64,000 & $\begin{array}{l}\text { Poot Delgado y Guzmán } \\
\text { Noz (2010) }\end{array}$ \\
\hline Leptocylindrus sp..$^{5}$ & \multirow{3}{*}{$\begin{array}{l}\text { Región centro litoral } \\
\text { (costa de Campeche- } \\
\text { Champotón) }\end{array}$} & \multirow{3}{*}{$\begin{array}{l}18 \text { septiembre al } 18 \\
\text { de octubre } 2009\end{array}$} & 870,000 & \multirow{3}{*}{ COPRISCAM y LESP* } \\
\hline Pseudo-nitzschia delicatissima? & & & 76,000 & \\
\hline Pyrodinium bahamense var. bahamense & & & 20,000 & \\
\hline Anabaena sp. ${ }^{3}$ & \multirow{2}{*}{ Litoral de Champotón } & Febrero de 2010 & 100,000 & \multirow{2}{*}{$\begin{array}{l}\text { Poot Delgado y Rosado } \\
\text { García (2013) }\end{array}$} \\
\hline Pyrodinium bahamense var. bahamense' & & Julio de 2010 & 311,000 & \\
\hline Oscillatoria erythraea? ${ }^{3}$ & Malecón de Campeche & 9 de agosto de 2010 & 36,000 & COPRISCAM Y LESP* \\
\hline \multirow{2}{*}{ Chaetoceros spp. ${ }^{4}$} & Litoral de Champotón & Noviembre de 2010 & $1,100,000$ & $\begin{array}{l}\text { Poot Delgado y Rosado } \\
\text { García (2013) }\end{array}$ \\
\hline & \multirow{3}{*}{$\begin{array}{l}\text { Region centro } \\
\text { litoral (Campeche- } \\
\text { Champotón) }\end{array}$} & \multirow{3}{*}{$\begin{array}{c}05 \text { al } 26 \text { de agosto } \\
\text { de } 2010\end{array}$} & 196,666 & \multirow{3}{*}{ COPRISCAM Y LESP* } \\
\hline Peridinium quinquecorne ${ }^{5}$ & & & 88,000 & \\
\hline Prorocentrum mexicanum ${ }^{4}$ & & & 47,660 & \\
\hline Cylindrotheca closterium ${ }^{5}$ & $\begin{array}{c}\text { Sistemas fluviolagunares } \\
\text { Pom-Atasta y Palizada } \\
\text { del Este } \\
\end{array}$ & $\begin{array}{c}12,13 \text { de febrero de } \\
2011\end{array}$ & 525,000 & $\begin{array}{l}\text { Muciño Márquez et al. } \\
\text { (2014) }\end{array}$ \\
\hline Karenia brevis?! & \multirow{2}{*}{$\begin{array}{l}\text { Región norte litoral } \\
\text { (Calkini, Hecelchakan, } \\
\text { Tenabo, Campeche y } \\
\text { Champotón) }\end{array}$} & \multirow{2}{*}{$\begin{array}{c}15 \text { al } 28 \text { de } \\
\text { septiembre } 2011\end{array}$} & $3,992,000$ & \multirow{2}{*}{ COPRISCAM Y LESP* } \\
\hline Pseudo-nitzschia spp.' & & & 50,000 & \\
\hline Prorocentrum mexicanum ${ }^{4}$ & \multirow{2}{*}{$\begin{array}{c}\text { Banco ostrícola (Laguna } \\
\text { de Términos) }\end{array}$} & Marzo de 2012 & 150,000 & \multirow{2}{*}{$\begin{array}{l}\text { Poot Delgado et al. } \\
\qquad(2015 a)\end{array}$} \\
\hline Gymnodinium sp.' & & Abril de 2012 & 150,000 & \\
\hline Karenia spp.' & Banco de Campeche & $\begin{array}{l}9 \text { de septiembre de } \\
2012\end{array}$ & $3,000,000$ & Soto Ramos et al. (2012) \\
\hline Cylindrospermopsis cuspis ${ }^{3}$ & $\begin{array}{c}\text { Banco ostrícola (Laguna } \\
\text { de Términos) }\end{array}$ & $\begin{array}{l}18 \text { de octubre de } \\
2012\end{array}$ & $10,860,000$ & COPRISCAM Y LESP* \\
\hline
\end{tabular}


Continuación de la Tabla 1

\begin{tabular}{|c|c|c|c|c|}
\hline \multirow{2}{*}{ Prorocentrum minimum² } & $\begin{array}{c}\text { Bancos ostrícola (Laguna } \\
\text { de Términos) }\end{array}$ & Noviembre de 2012 & $2,300,000$ & $\begin{array}{l}\text { Poot Delgado et al. } \\
\text { (2015a) }\end{array}$ \\
\hline & $\begin{array}{l}\text { Muelle la Puntilla, Ciudad } \\
\text { del Carmen }\end{array}$ & $\begin{array}{l}\text { Octubre de } 2012 \text { a } \\
\text { enero de } 2013\end{array}$ & $2,363,000$ & $\begin{array}{l}\text { Poot Delgado et al. } \\
\qquad(2015 b)\end{array}$ \\
\hline Pseudo-nitzschia spp.' & \multirow{3}{*}{$\begin{array}{c}\text { Banco ostrícola (Laguna } \\
\text { de Términos) }\end{array}$} & Enero de 2013 & 640,000 & \multirow{3}{*}{$\begin{array}{l}\text { Poot Delgado et al. } \\
(2015 b)\end{array}$} \\
\hline Cylindrotheca closterium ${ }^{5}$ & & Febrero de 2013 & 200,000 & \\
\hline Anabaena sp. ${ }^{3}$ & & Mayo de 2013 & 660,000 & \\
\hline Cyclotella sp. ${ }^{5}$ & \multirow{2}{*}{$\begin{array}{l}\text { Muelle la Puntilla, Ciudad } \\
\text { del Carmen }\end{array}$} & Agosto de 2013 & $6,500,469$ & \multirow{2}{*}{$\begin{array}{l}\text { Poot Delgado et al. } \\
\qquad(2015 b)\end{array}$} \\
\hline Peridinium quinquecorne ${ }^{5}$ & & Septiembre de 2013 & $1,532,771$ & \\
\hline Rhizosolenia sp. ${ }^{5}$ & Litoral de Champotón & Noviembre de 2013 & 180,000 & $\begin{array}{l}\text { Rendón von Osten et al. } \\
\qquad(2013)\end{array}$ \\
\hline
\end{tabular}

* Comisión para la Protección y Riesgo Sanitario del estado de Campeche. Laboratorio Estatal de Salud Pública del Estado de Campeche.

'Especie conocida como potencialmente tóxica: causante de Intoxicación paralítica por mariscos (PSP) (IOC-UNESCO, 2002).

${ }^{2}$ Responsable de mortalidad en bioensayo ratón, a través de una toxina soluble en agua. Sin embargo, no hay datos que indican los impactos a los consumidores humanos (Grzebyk et al., 1997).

${ }^{3}$ Especies conocidas como potencialmente tóxicas: microcistina LR, lipopolisacáridos (LPS), microcistina, anatoxina-a, anatoxina-a (S), saxitoxina, cilindrospermopsina, neusaxitoxina y neosaxitoxina (Bonilla, 2009).

${ }^{4}$ Especies que se sabe o se sospecha que causan pérdidas de peces en maricultura (Anderson et al., 2001).

${ }^{5}$ Especies que se sabe producen florecimientos en otros lugares (Anderson et al., 2001) o cuya abundancia de vez en cuando alcanza concentra-

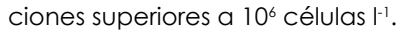

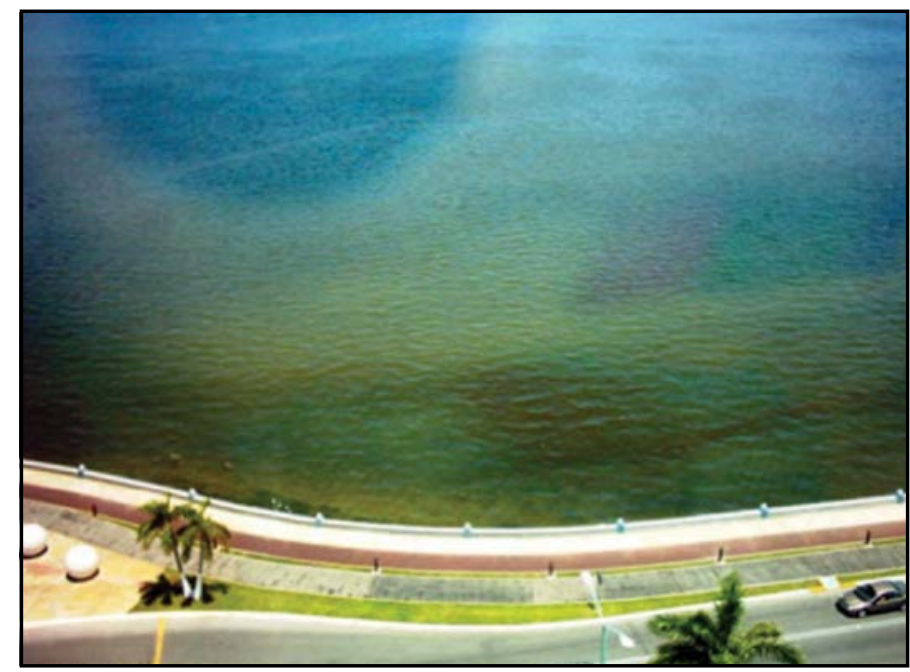

Figura 2. Florecimiento de $P$. quinquecorne en el malecón de la ciudad de San Francisco de Campeche. Fotografía cortesía de Fausto Tafoya.

En febrero de 2011 Muciño Márquez et al. (2014) presentaron resultados de los sistemas fluviolagunares Pom-Atasta y Palizada del Este, en donde registran que Cylindrotheca closterium fue la especie más abundante en los dos sistemas, con

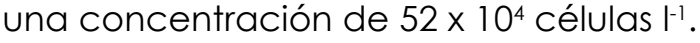

Por otro lado, Poot Delgado et al. (2015a) reportaron la abundancia de fitoplancton y su variación estacional de julio de 2012 a septiembre de 2013. Se registraron valores mínimos $\left(10^{3}\right.$ células
$1^{-1}$ ) durante la época de secas (febrero-mayo) y valores altos $\left(10^{6}\right.$ células $\left.^{-1}\right)$ durante la temporada de lluvias (junio-septiembre), así como la presencia de especies de dinoflagelados nocivos: Akashiwo sanguinea, K. mikimotoi, P. bahamense var. bahamense, $P$. mexicanum y $P$. minimum. También la presencia de los géneros Alexandrium y Pseudonitzschia (ver Tabla 1).

Recientemente Núñez Vázquez et al. (2013) reportaron para la zona central de Campeche la presencia de análogos de toxinas paralizantes (dcGTX2-3, GTX2-3, NeoSTX y STX) que se asemejan al perfil de toxinas descrito para el dinoflagelado $P$. bahamense (Landsberg et al., 2006), que de acuerdo con lo reportado previamente por Poot Delgado et al. (2014), no rebasa el orden de $10^{4}$ células $~^{-1}$. Asimismo, se ha reportado que otras biotoxinas marinas (amnésicas y diarreicas) han afectado a peces (Sphoeroides), las cuales tienen su origen en las especies planctónicas, dado que en las costas de Campeche se han detectado florecimientos de microalgas nocivas (Figura 2), productoras de toxinas tales como P. bahamense, Pseudo-nitzschia y Dinophysis (Poot Delgado y Rosado García, 2013) que pueden ser transmitidas a los peces Sphoeroides a través de la cadena alimentaria vía moluscos bivalvos y otros invertebrados, organismos que regularmente forman parte de la dieta de estos peces en el litoral campechano (Mallard Colmenero et al., 1982). 


\section{Cianobacterias nocivas}

En años recientes ha aumentado la literatura que menciona que los cuerpos de agua costeros tienen altas abundancias de cianobacterias $\left(10^{6}\right.$ células $\left.{ }^{-1}\right)$, particularmente durante la época de secas (febrero-mayo), pero su contribución a la biomasa total del fitoplancton es a menudo relativamente pequeña (Pinckney et al., 1998; Ning et al., 2000).

Se sabe que algunas especies producen toxinas capaces de causar efectos agudos y crónicos en el hombre, en animales y vegetales (Landsberg, 2002). Se estima que más de $50 \%$ de estas proliferaciones son tóxicas (Codd et al., 1999).

Las toxinas de las cianobacterias se suelen agrupar principalmente en neurotoxinas y hepatotoxinas. Las neurotoxinas son producidas principalmente por especies y cepas de los géneros: Anabaena, Aphanizomenon (Mahmood y Carmichael, 1986), Oscillatoria (Sivonen et al., 1989), Trichodesmium (Hawser et al., 1991) y Cylindrospermopsis (Landsberg, 2002). Las especies identificadas como productoras de hepatoxinas están incluidas en los géneros Microcystis, Anabaena, Nodularia, Oscillatoria, Nostoc y Cylindrospermopsis
(Landsberg, 2002). El género Anabaena ha estado presente a lo largo del litoral campechano, desde la bahía de Campeche hasta los bancos ostrícolas localizados en la Laguna de Términos, con

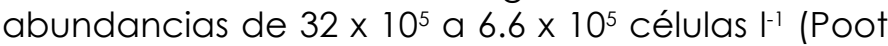
Delgado et al., 2015b). Sin embargo, en octubre de 2012 también se registró a Cylindrospermopsis cuspis, con una abundancia de $1.3 \times 10^{6}$ células $1^{-1}$, que perduró hasta noviembre de 2012 con un valor

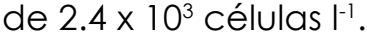

\section{CONCLUSIONES}

Este análisis muestra que ha habido un avance significativo en los reportes de FAN en las costas campechanas por parte de la autoridad competente y académicos; sin embargo, se observa que su estudio se ha abordado de manera individualizada, con diferentes métodos y de forma dispersa.

\section{Agradecimientos}

Al Mtro. Jorge A. Ortiz Lozano por la elaboración del mapa de localización, al Dr. Yuri Okolodkov y a Marcia M. Gowing, que amablemente mejoraron el resumen y el abstract. A los revisores anónimos por las sugerencias para mejorar el manuscrito.

\section{LITERATURA CITADA}

- ALONSO RODRÍGUEZ, R. y PÁEZ OSUNA, F. Nutrients, phytoplankton and harmful algal blooms in shrimp ponds: a review with special reference to the situation in the Gulf of California. Aquaculture, 219(1-4): 317-336, 2003.

- ANDERSON, D. M. et al. Monitoring and management strategies for harmful algal blooms in coastal waters. Asia Pacific Economic Program, Singapore, and Intergovernmental Oceanographic Commission Technical Series, 59, 1-268, 2001.

- ANDERSON, D. M. et al. Progress in understanding harmful algal blooms: paradigm shifts and new technologies for research, monitoring, and management. Annual Review of Marine Science, 4, 143-176, 2012.

- BAND SCHMIDT, C. J. et al. El estado actual del estudio de florecimientos algales nocivos en México. Hidrobiológica, $21(3): 381-413,2011$.

- BONILLA, S. (Ed.). Cianobacterias planctónicas del Uruguay. Manual para la identificación y medidas de gestión. Documento Técnico PHI 16. Programa Hidrológico Internacional para América Latina y el Caribe (PHI-LAC). Oficina Regional de Ciencia para América Latina y el Caribe. Montevideo, Uruguay: UNESCO, 2009.
- BRICKER, S. et al. An integrated methodology for assessment of estuarine trophic status. Ecological Modelling, 169(2003): 39-60, 2003.

- CODD, G. et al. Cyanobacterial toxins, exposure routes and human health. European Journal of Phycology, 34 (4): 405-415, 1999.

- DEL ÁNGEL TAFOYA, F. 10 años de monitoreo en la atención de florecimiento algal en el estado de Campeche. Reunión de responsables nacionales y estatales de los temas de moluscos bivalvos y marea roja. Comisión de Operación Sanitaria. Dirección Ejecutiva de Programas Especiales. Ensenada, Baja California, México, del 8 al 12 de julio de 2013.

- gÁRATE lizÁRRAGA, I. y GONZÁleZ ARMAS, R. Fish die-offs along southern coast of Baja California. Harmful Algae News, $48,8-9,2014$.

- GliBERT, P. M. et al. The global, complex phenomena of harmful algal blooms. Oceanography, 18(2): 136-147, 2005.

- GRZEBYK, D. et al. Evidence of a new toxin in the red-tide dinoflagellate Prorocentrum minimum. Journal of Plankton Research, 19(8): 1111-1124, 1997. 
- HAWSER, S. P. et al. A neurotoxic factor associated with the bloom-forming cyanobacterium Trichodesmium. Toxicon, 29(3): 277-278, 1991.

- KAAS, H. et al. Marine phytoplankton: Relationship between ecological and chemical status of surface waters. REBECCA SSPI-CT-2003-502158 7-10, 2005.

- LANDSBERG, J. H. The effects of harmful algal blooms on aquatic organisms. Reviews in Fisheries Science, 10(2): 113390, 2002.

- LANDSBERG, J. H. et al. Saxitoxin puffer fish poisoning in the United States, with the first report of Pyrodinium bahamense as the putative toxin source. Environmental Health Perspective, 114(10): 1502-1507, 2006.

- MAHMOOD, N. y CARMICHAEL, W. Paralytic shellfish poisons produced by the freshwater cyanobacterium Aphanizomenon flos-aquae NH-5. Toxicon, 24, 175-186, 1986.

- MALLARD COLMENERO, L. R. et al. Taxonomía, biología y ecología de los tetraodóntidos de la Laguna de Términos, Sur del Golfo de México (Pisces: Tetraodontidae). Anales del Instituto de Ciencias del Mar y Limnología, 9(1): 161-211, 1982.

- MUCIÑO MÁRQUEZ, R. et al. Composición fitoplanctónica en los sistemas fluvio-lagunares Pom-Atasta y Palizada del Este, adyacentes a la Laguna de Términos Campeche, México. Acta Biológica Colombiana, 19(1): 63-84, 2014.

- NING, X. et al. Spatial and temporal variability of picocyanobacteria Synechococcus sp. in San Francisco Bay. Limnology and Oceanography, 45(3): 695-702, 2000.

- NÚÑEZ VÁZQUEZ, E. et al. Impact of harmful algal blooms on wild and culture animals in the Gulf of California. Journal of Environmental Biology, 32(4): 413-423, 2011.

- NÚÑEZ VÁZQUEZ, E. et al. Toxicidad de los botetes silvestres Sphoeroides spp., y Lagocephalus spp., de las costas de Campeche, México. Memorias del XX Congreso Nacional de Ciencia y Tecnología del Mar. Los Cabos, B. C. S., del 1 al 5 de octubre de 2013.

- OKOLODKOV, Y. B. A review of Russian plankton research in the Gulf of Mexico and the Caribbean Sea in the 1960-1980s. Hidrobiológica, 13(3): 207-221, 2003.

- PAERL, H. et al. Anthropogenic and climatic influences on the eutrophication of large estuarine ecosystems. Limnology and Oceanography, 51 (1_part_2): 448-462, 2006.

- PINCKNEY, J. et al. Annual cycles of phytoplankton communitystructure and bloom dynamics in the Neuse River Estuary, North Carolina. Marine Biology, 131 (2): 371-381, 1998.

- POOT Delgado, C. A. y GUZMÁN NOZ, Y. Composición y abundancia del fitoplancton marino, con énfasis en las especies potencialmente tóxicas y/o nocivas, en la Bahía de Campeche, México. Memorias del III Congreso Internacional de Investigación. Parral, Chihuahua, del 13 al 15 de octubre de 2010.

- POOT DELGADO, C. A. y ROSADO GARCíA, P. Fitoplancton marino potencialmente nocivo en las aguas costeras de Champotón, Campeche. Memorias del XX Congreso Nacional de Ciencia y Tecnología del Mar. Los Cabos, B. C. S., del 1 al 5 de octubre de 2013.

- POOT DELGADO, C. A. et al. Annual cycle of phytoplankton with emphasis on potentially harmful species in oyster beds of Terminos Lagoon, southeastern Gulf of Mexico. Revista de Biología Marina y Oceanografía, 50(3): 465-477, 2015a.

- POOT DELGADO, C. A. et al. Fitoplancton marino potencialmente nocivo en las aguas costeras de Campeche. En A.V. Botello, J. Rendón von Osten, J. A. Benítez y G. Gold Bouchot (Eds.), Golfo de México. Contaminación e impacto ambiental: diagnóstico y tendencias (117-132). UAC, UNAM-ICMYL, CINVESTAV-Unidad Mérida, 2014

- POOT DELGADO, C. A. et al. Fitoplancton potencialmente nocivo en el muelle La puntilla, Laguna de Términos, sureste del Golfo de México. BIOCYT Biología, Ciencia y Tecnología, 8(32): 570-582, 2015b.

- POOT DELGADO, C. et al. Primer reporte de Pyrodinium bahamense var. bahamense (Gonyaulacales: Goniodomataceae), dinoflagelado tóxico, en la Bahía de Campeche, México. Memorias del XVI Congreso Nacional de Ciencia y Tecnología del Mar. Colima, Colima, México, del 5 al 7 de octubre de 2009.

- RENDÓN VON OSTEN, J. et al. Florecimientos algales nocivos (FAN): eventos que pueden matar ballenas. JAINA, 25(2): 5-10, 2013.

- SAR, E. A. et al. (Eds.). Floraciones algales nocivas en el Cono Sur Americano. Madrid, España: Instituto Español de Oceanografía, 303 pp., 2002.

- SIVONEN, K. et al. Preliminary characterization of neurotoxic cyanobacetrial blooms and strains from Finland. Toxicity Assessment, 4, 339-352, 1989.

- SMAYDA, T. Harmful algal blooms: their ecophysiology and general relevance to phytoplankton blooms in the sea. Limnology and Oceanography, 42(5-II): 1137-1153, 1997.

- SOTO RAMOS, I. et al. Binational collaboration to study Gulf of Mexico's harmful algae.EOS, Transactions American Geophysical Union, 93(5): 49-50, 2012

\section{De páginas electrónicas}

- IOC-UNESCO (Intergovernmental Oceanographic Commission of UNESCO). Taxonomic reference list of harmful micro algae. 2002. Recuperado el 30 de septiembre de 2015, de http://www. marinespecies.org/hab/ 